\title{
Theoretical Study of Movements Trash in Cotton Lobules In Cleaner Small and Large Trash
}

\author{
Egamnazar Gaibnazarov ${ }^{1}$, Botir Mardonov ${ }^{2}$, Azizov Shuhrat Mamatovich ${ }^{1 *}$ and Shaxboz Isayev ${ }^{1}$ \\ ${ }^{1}$ Namangan Institute of Engineering and Technology, Russia \\ ${ }^{2}$ Tashkent Institute of Textile and Light Industry of Uzbekistan, Russia
}

Received: January 02, 2018; Published: January 18, 2018

*Corresponding author: Azizov Shuhrat Mamatovich, Namangan Institute of Engineering and Technology, Uzbekistan, Russia

\section{Abstract}

The article considered mathematical model that describes the separation of large and small trash by weight of cotton, with elastic connection on the cotton. The relationship between the forces acting on the speck on the angular velocity and the radius of the working drum cleaner, laws of motion specks obtained on the surface and inside the cotton mass on the process parameters.

Keywords: Pulp; Speck; Elastic element; The force of the bunch; The dry friction force; Kolkova drum; Surface netted; The angular velocity

\section{Introduction}

Known designs of cleaners are based on shock-shaking effects on fibrous material [1]. Despite the fact that improving the design of cleaners has an ever-increasing trend, the industry's demand for highly efficient cleaners does not decrease. It should also be noted that, with the improvement of the designs of cleaners, the basic working elements of them remain the same. This, in our opinion, is a consequence of the fact that the essence of the process of cleaning fibrous material from weedy impurities is not sufficiently disclosed, the available dynamic and mathematical models do not fully correspond to the actual cleaning situation. Therefore, any progressive attempts to describe the purification process using the latest achievements of science should be welcomed. Linear [2], nonlinear [3] dynamic models of the process for cleaning fibrous material are known. Also developed are models aimed at isolating weedy impurities from the surface of a layer of fibrous material [4], isolating weed particles from the fibrous material by shearing the layers [5], isolating the litter during their interaction with the working organs of the disintegrated [6].

There are also attempts to describe the process by empirical formulas based on experimental material [7]. Despite the fact that the models adequately solve the tasks assigned to them, due to accepted assumptions in the development or in the analysis of models, some questions were not answered, which served as the development of a dynamic model that takes into account the masses of the mote associated with fibrous material by an elastic bond, interacting with the surface of the material by the force of dry friction. Let the fibrous mass contain a mote with a mass that is connected to it by an elastic element and by the force of dry friction. Depending on the location in the composition of the pulp, we divide the trash into two types. The first type of trash is located on the surface of the scrap and is in contact with the mesh surface. Such mote has an angular velocity equal to the speed of the spike, and in addition, under the action of a centrifugal force, they preferentially move through the opening of the mesh in the radial direction from the center.

The distance from the center to the mote will be denoted by, we assume that the mote is connected to the fibrous mass through an elastic element with variable rigidity and write down the equation of motion of the mote.

$$
m_{c} \ddot{r}=m_{c} g \sin (\alpha+\omega t)+m_{c} \dot{r}^{2} / R-k_{c}(r-R)
$$

Where - coefficient of stiffness of a bundle of a mote with fibers and depends on the displacement $r-R$, т.e. $k_{c}=k_{c}(r-R)$ , where function $k_{c}(z)$ is determined experimentally and has a monotonically decreasing character, and after detachment of the mote from the fibrous mass, it vanishes, i.e. should be taken $k_{c}(z)=0$ at $z>z_{0}$. In particular, this function can be chosen in the form of a piecewise constant $k=k_{0}$ at $z<z_{0}$ and $k=0$ at $z>z_{0}$ or piecewise linear $k_{c}=k_{1}\left(z_{0}-z\right)$ at $z \leq z_{0}$ and $k=0$ at $z \geq z_{0}$ Moving $r(t)$ satisfies the following initial conditions $r=R$, $\dot{r}=R \omega_{a}^{2}$ at $t=0$. In this case, the leaving time $t=t_{k}$ the mote of the mesh surface is determined from equations:

$$
k\left[r\left(t_{k}\right)-R\right]=0
$$


If $t_{0} \leq t_{k}$ then the mote has time to leave the surface of the grid during the contact of the scrap with the surface of the grid, but if $t_{0}>t_{k}$, then the mote after the interaction of the scrap with the reticulate surface does not leave the scrap and continues to move with it. The results of integrating equations (1) are presented in Figure 1. The second type includes the mote, which is in the composition of the pulp at a distance from the center of the drum, and $r_{1}<R$. In this case, these kinds of trash move for some time in the region within the fibrous mass, the movement of the trash relative to the scrap preferably in the radial direction [8-10]. To compose the equation of motion of the mote, we assume that the force of the bond between the mote and fibers acts on the mote, and also the frictional force at the surface of their contact as a result of the movement of the mote relative to the fibrous mass.

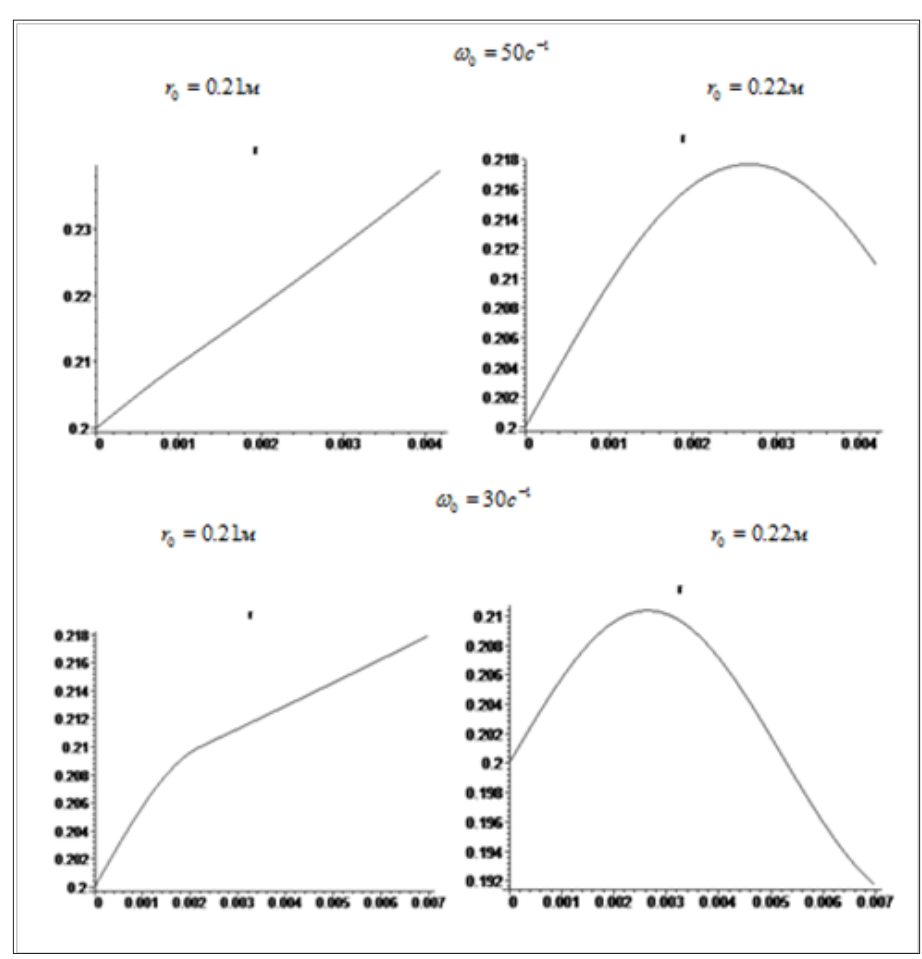

Figure 1: Obligations of radial displacement mote for time for different values of movement angular velocity of the drum.

$(x, y)$ (in the plane $x 0 y$.

$x=r(t) \sin \theta, \quad y=r(t) \cos \theta$

Let's compose the equations of kinetic energy of a mote

$$
T=\frac{m_{c}}{2}\left(\dot{x}^{2}+\dot{y}^{2}\right)=\frac{m_{c}}{2}\left(\dot{r}^{2}+r^{2} \dot{\theta}^{2}\right)
$$

The following forces act on the mote with projections along the axes и

a) Gravity of mote

$X_{1}=0, \quad Y_{1}=-m_{c} g$

b) Force of a bundle of a mote with fibers, depending on distance

$$
X_{2}=-k_{c}(r-R)(r-R) \sin \theta, Y_{2}=-k_{c}(r-R)(r-R) \cos \theta
$$

c) The force of dry friction on the contact surface of the mote with pulp

$X_{3}=-m g f \frac{\dot{x}}{\sqrt{\dot{r}^{2}+r^{2} \dot{\theta}^{2}}}, \quad Y_{3}=-m g f \frac{\dot{y}}{\sqrt{\dot{r}^{2}+r^{2} \dot{\theta}^{2}}}$

We take the coordinate

$r$ as a generalized one and find the generalized force.

$$
Q_{r}=\left(X_{1}+X_{2}+X_{3}\right) \frac{\partial x}{\partial r}+\left(Y_{1}+Y_{2}+Y_{3}\right) \frac{\partial y}{\partial r}
$$

Using the expressions

(3.12.4) - (3.12.6), we get

$Q_{r}=-k_{c}(r-R)(r-R)-\frac{m g f \dot{r}}{\sqrt{\dot{r}^{2}+r^{2} \dot{\theta}^{2}}}-m g \cos \theta$

Let us now compose the Lagrange equation of II -three,

$\frac{d}{d t}\left(\frac{\partial T}{\partial \dot{r}}\right)-\frac{\partial T}{\partial r}=Q_{r}$

By supplying the expressions for the kinetic energy (3) and the generalized force (7), we form the equation for the definition of the variable $r$.

$m_{\dot{a}} \ddot{r}=m_{c} \dot{\theta}^{2} r^{2}-k_{c}(r-R)(r-R)-\frac{m_{\dot{n}} g f \dot{r}}{\sqrt{\dot{r}^{2}+r^{2} \dot{\theta}^{2}}}+m g \sin \theta$

Equation (8) with the known angular displacement of the scrap.

$\theta=\theta(t)$

Integrates under the initial conditions.

$r=R, \dot{r}=R \omega_{\dot{a}}$ at $t=0$

On Figure 2 the curves of the distance dependence $r(t)$ (M) and the rate of moth removal $\dot{r}(t)(\mathrm{M} / \mathrm{c})$ in the case when $\theta=\omega_{b} t$ 
, $k_{c}=k_{c 0}=$ const Calculations were made for different values of the parameter $\omega_{* *}=\sqrt{k_{c} / m_{c}}(1 / c e k)$. With small masses of mote, this value can assume large values. Therefore, calculations were

carried out for $\omega_{* *}>5001 /$ cek. The calculations also $R=0.2$, $\omega_{b}=801 / c e k, f=0.3, \theta_{0}=20^{\circ}, \sqrt{k / m}=801 / c e k$.

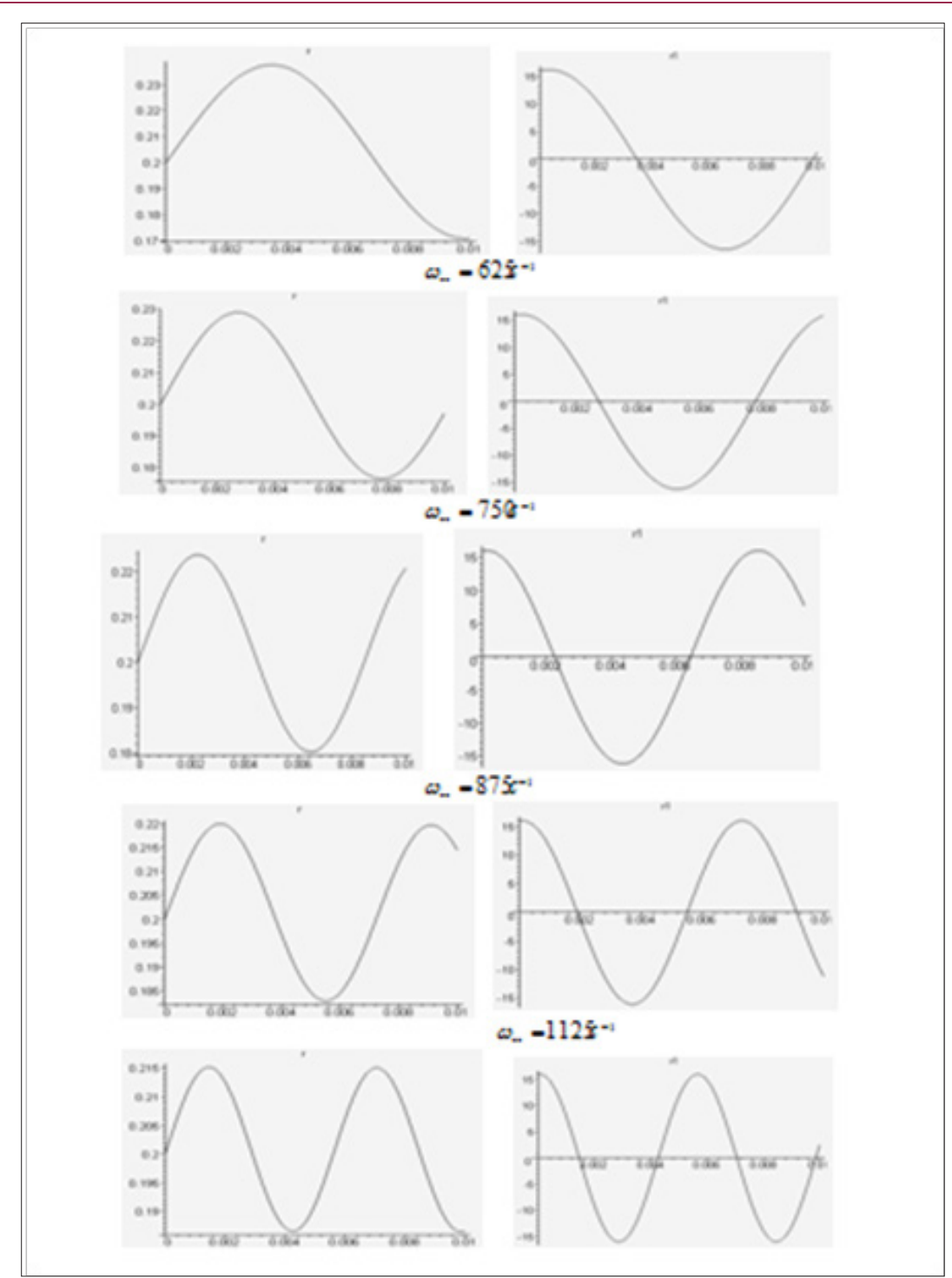

Figure 2: Dependence of displacement $r(t)$ and speed $r_{1}=\dot{r}(\mathrm{e})$ of time for different values of the parameter $\omega_{* *}\left(c^{-1}\right)$.

It can be seen from the results that this parameter plays a significant role in the process of removing the mote [11]. At small values, the mote is rapidly removed from the scrap, for example, if it is assumed that the detachment of the mote from the scrap occurs when the stretch $\delta=0.02$, then the weight of the mote for which the parameter $\omega_{* *}$ takes on values $\omega_{* *}<1000$ then they are removed from the pulp. Other specks where $\omega_{* *}>1000$, then the initial velocity of the mote will be insufficient to remove from the mass. If the speed of the drum is taken $\omega_{i}=120 \tilde{n} a e^{-1}$ then specks with a parameter $\omega_{* *}=1000$ will also be deleted.

\section{Conclusion}

As a result of the analysis of the dynamic model for the interaction of weed impurities with the pulp with an elastic coupling between them, the dependency of the parameters of the displacement of the rubbish on the angular velocity and the radius of the cleaning drum of the cleaner is established, which can be used in the development of purifiers for raw cotton.

\section{References}

1. (2008) Handbook of primary cotton processing. Edited by Omonov FB (in the Uzbek language). Asia.

2. (1972) Miroshnichenko GI Basics of designing machines for primary cotton processing. M Mechanical Engineering pp. 486.

3. Lebedev DA (2009) A nonlinear model of the effect on a particulate particle during fiber cleaning. Magazine Technology of the textile industry 5: 320 .

4. Korabelnikov AR (2012) Isolation of impurities from the surface of a layer of fibrous material. Magazine Technology of the textile industry 4 : 340 . 
5. Korabelnikov AR, Korabelnikov RV, Lebedev DA (2010) Theoretical aspects of the mechanism of separation of weed particles from fibrous material when shearing layers (discharge). Magazine Technology of the textile industry 8: 329 .

6. Khosrovyan IG (2012) A general theory of the dynamics of fibrous complexes in the process of their interaction with the working organs of a disintegrant. Magazine Technology of the textile industry 6: 342

7. Sevostyanov AG (1980) Methods and means of studying the mechanical and technological processes of the textile industry. Moscow «Light industry». Recommended by the Department of Technology of Primary Processing of Natural Fibers of the Namangan Engineering and Technology Institute.

8. Mamatovich A Sh, Axmedhodjaev XT (2015) Theoretical Analysis of Gin Cylinder for Simulating Dual Saw Cylinder Chamber Gin for Increasing
Wear proof, Energy Efficient, Saving Resources. World Journal of Engineering and Technology 3(3): 8.

9. Shuhrat Mamatovich Azizov, Xamit Tursunovich Axmedhodjaev (2016) The Optimal Modeling of an Angular Position of Saw Cylinders in SingleChamber Two Cylinders Gin. American Journal of Mechanical and Industrial Engineering 1(3): 103-106.

10. Mamatovich A Sh, Abdusamat K, Arras P (2013) The Mathematical Simulation of Brush Drums in a Dual Saw Cylinder Chamber Gin for the Purpose of Increasing the Quantity of Captured Cotton Fiber from Saw. World Journal of Mechanics 3(1): 58-61.

11. Mamamtovich AS (2016) Analysis of the Influence of Geometric Characteristics of the Saw and the Gasket of Saw Gin on the Life of Saw at Different Distances between the Saw. J Textile Sci Eng 6: 256.

DOI: $10.32474 /$ LTTFD.2018.01.000101

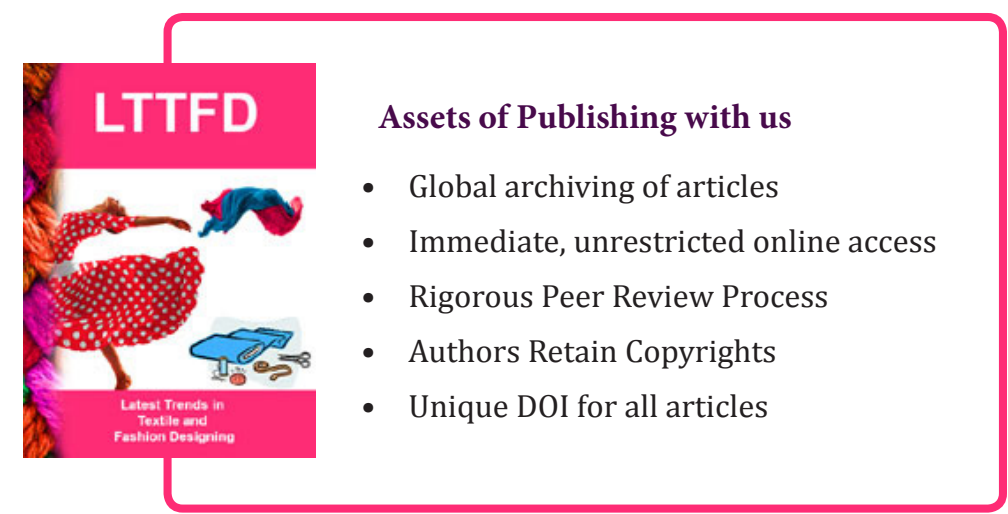

